www.jmscr.igmpublication.org

Impact Factor 5.84

Index Copernicus Value: 71.58

ISSN (e)-2347-176x ISSN (p) 2455-0450

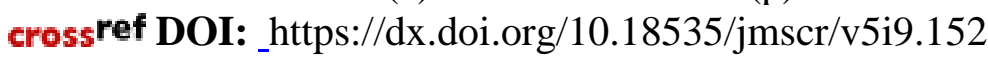

Journal Of Medical Science And Clinical Research

\title{
A Study on Assessment of Health Related Quality of Life and Treatment Satisfaction in type 2 Diabetic Patients
}

\author{
Authors \\ MD Tarique Nadeem ${ }^{1}$, Vikram.P ${ }^{2}$, Divya Sri. B ${ }^{3}$, Vijaya.K $^{4}$, MD. Azharuddin ${ }^{5}$ \\ ${ }^{1}$ Dept of Endocrinoloy, Jawaharlal Institute of Post Graduate Medical Education and Research, Pondicherry \\ ${ }^{2,3,4}$ Pharm. D Smt. Sarojini Ramulamma College of Pharmacy, Mahabubnagar \\ ${ }^{5}$ Department of Pharmaceutical Medicine, Hamdard University, New Delhi
}

Corresponding Author

MD.Tarique Nadeem

Dept of Endocrinoloy, Jawaharlal Institute of Post Graduate Medical Education and Research, Pondicherry

Tel: 9128171518, Email: tan.jipmer2017@ gmail.com

\begin{abstract}
Introduction: Type 2 diabetes which currently is one of the most important chronic diseases in the world reduces the quality of life in patients.

Materials and Methods: Our research as a descriptive analytical observational study was performed on 103 type 2 Diabetes Mellitus patients in Mahabubnagar (INDIA). Diverse domain scores of patients (HRQOL), well-being index and treatment satisfaction were evaluated by SF-36standard questionnaire, WHO(5) well being index and diabetes treatment satisfaction questionnaire(DTSQ). Gained data was analysed by using SPSS 16.0 software through statistical tests including independent $T$ test, one-way ANOVA and sample T- test.
\end{abstract}

Results: The type 2 diabetic population has a quality of life score of more than 50 in most of the survey aspects. We found the lowest scores for the aspect of "role physical"(25). Extremely high scores were found for the aspects: "social function"(71.50) and "role emotional"(60.92). Statistically different observations were found between men and women for "social function" $(P=0.002)$. Age had significant reverse relationship with physical functioning, vitality, and mental component score. The WHO well-being score for men (58.23) and women (75.05). 50\%of patients scored 33 or higher for diabetes treatment satisfaction questionnaire.

Conclusion: Type 2 diabetes mellitus is associated with poor self-perceived health-related quality of life (HRQOL).Taken together, the findings of this population studies indicate that people using either pharmacological therapy or lifestyle modification to treat diabetes rated their physical health as poor.

Keywords: Type 2 Diabetes, Health Related Quality Of Life (HRQOL), Diabetes Treatment Satisfaction Questionnaire (DTSQ).

\section{Introduction}

Diabetes Mellitus is a metabolic disorder characterised by chronic hyperglycaemia with impaired carbohydrate, fat and protein metabolism, which results in defects of inslin secretion, insulin action, or both. It has characteristic symptoms like thirst, polyuria, blurred vision and weight loss ${ }^{[1]}$. 
Common complications of Diabetes are retinopathy, nephropathy, neuropathy, cardiovascular diseases and infections. Diabetes is a serious health problem which threats the patients' quality of life. So it seems to be important to study the patient's quality of life. Complications may further affect quality of life in diabetes in patients $[2,3,4]$.

WHO defines quality of life as individuals perception of their position in life in the context of the culture and value systems in which they live and in relation to their goals, expectations, standards and concerns. It is a board ranging concept affected in a complex way by the persons physical health, psychological state, level of independence, social relationships, personal beliefs and their relationship to salient features of their environment ${ }^{[5]}$.

Health related Quality of Life (HRQOL) is "a multidimensional concept referring to a person's total well-being, including his or her psychological, social, and physical health status ${ }^{[6]}$.

The aim of modifiedSF-36 questionnaireis to describe the health-related quality of life in people with diabetes, compared to those without diabetes. It measures quality of life which profiles eight domains namely Physical Functioning (PF), Role Physical (RP), Bodily Pain (BP), General Health (GH), Vitality (VT), Social Functioning (SF), Role Emotional (RE), Mental Health (MH). Higher scores indicate better health or level of functioning ${ }^{[7,8]}$.

Using a short questionnaire of WHO-5 can help to monitor emotional well-being in patients as part of clinical routine and enhance the likelihood of recognizing depression ${ }^{[9]}$.

We tried to measure quality of life in type 2 diabetes patients and identify related effective factors in it.

\section{Material and Methods}

This research- is a prospective observational study conducted at a 300 bedded super speciality teaching hospital at Mahabubnagar. The study was conducted for a period of six months and the study was approved by approved by the Institutional Ethical Committee (IEC) of the hospital ref.id. SVSMC/IEC/2016/47(4). Sample size was 103 patients.

We evaluated diverse domains of diabetic patients quality of life scores through SF-36 standard questionnaire composed of 36 questions. This questionnaire is one of most common tools for quality of life measurement. Validity and reliability of SF-36 questionnaire have been approved in several studies. The quality of life was also evaluated by WHO- Well Being Questionnaire, WHO- Diabetes Treatment Satisfaction Questionnaire and Likert type 7 point satisfaction scale.

We also measured some other personal characterristics including age, sex, body mass index (BMI), education level, family income level, smoking, alcoholism, c morbidity, family history.

Analyzing all the questionnaires, we tried to find their relations to diverse domains of patients' quality of life by SPSS16.0, graph pad prism software though statistical tests including One sample $\mathrm{t}$ test, Student $\mathrm{t}$ test (two tailed, independent) and One way ANOVA. Microsoft word and excel (2007) have been used to generate graphs, tables etc.

\section{Results}

A total of 103 people participated in the study with an average age of $50.16 \pm 0.81$. The majority of participants were between the age group of 46$55(43.7 \%)$. As we can observe from Table 1, women make up $49.5 \%$ of the sample study with 51 participants and men make up the remaining $50.5 \%$ with 52 participants. The youngest and oldest patients were 25 and 60 years old, respectively. Mean BMI of participants was 24.8 (SEM = 0.42). Minimum and maximum BMIs were 18 and 33, respectively. $52.4 \%$ of patients were illiterate and $47.6 \%$ literates. $83.5 \%$ of patients were non-smokers and $16.5 \%$ of patients were smokers. $74.8 \%$ of patients were nonalcoholic and $25.2 \%$ of patients were alcoholic. Co morbidity was present in $59.2 \%$ of patients and $40.8 \%$ were without Co morbidity [Table 1]. 
The resultant mean and standard error mean for diverse domain of patients quality of life (both genders) were as followed, respectively: Physical functioning 53.96 (2.32), role physical 25.00 (3.79), bodily pain 60.31 (2.83), general health 53.79 (1.88), vitality 55.02(1.93), social functioning 71.50 (1.97), role emotional 60.92 (1.96), mental health 41.10 (4.46), physical component score 48.27(1.51), mental component score 57.13 (1.92) [Table2]. Overall variables of the resultant mean and standard error mean for diverse.

Domain of patients quality of life can be seen from the [Table 3].

Our research showed that age variables had meaningful reverse relationship with physical functioning ( $\mathrm{P}=0.043)$, vitality $(\mathrm{P}=0.007)$, physical component score $(\mathrm{P}=0.054)$, mental component score $(\mathrm{P}=0.003)$ domains. There was no relationship between age and other quality of life domains [Table 3,4].

Gender correlated to bodily pain $(\mathrm{P}=0.085)$, social functioning $(\mathrm{P}=0.002)$, role emotional $(\mathrm{P}$ $=0.011)$ physical component score $(\mathrm{P}=0.064)$, mental component score $(\mathrm{P}=0.042)$ as women score was higher than men. There was no relationship between gender and other quality of life domains.

Education level had direct correlation with role physical $(\mathrm{P}=0.016)$, general health $(\mathrm{P}=0.019)$, social function $(\mathrm{P}=0.007)$, role emotional $(\mathrm{P}=$ 0.054), mental health $(\mathrm{P}=0.003)$, mental component score $(\mathrm{P}=0.008)$. There was no relationship between education level and other quality of life domains.

Smoking status correlated with bodily pain $(\mathrm{P}=$ 0.007), physical component score $(\mathrm{P}=0.007)$. There was no relationship between smoking status and other quality of life domains.

Alcoholic correlated with bodily pain $(\mathrm{P}=0.007)$, general health $(\mathrm{P}=0.012)$, vitality $(\mathrm{P}=0.050)$, social functioning $(\mathrm{P}=0.003)$, role emotional $(\mathrm{P}=$ $0.029)$ physical component score $(\mathrm{P}=0.013)$. There was no relationship between alcoholic and other quality of life domains.
No relation was found between co morbidity's and other quality of life domains.

WHO well-being had direct correlation with bodily pain $(\mathrm{P}=0.000)$, general health $(\mathrm{P}=$ $0.000)$, vitality $(\mathrm{P}=0.003)$, social functioning ( $\mathrm{P}$ $=0.001)$, role emotional $(\mathrm{P}=0.001)$, mental health $(\mathrm{P}=0.024)$, physical component score $(\mathrm{P}=$ $0.002)$ mental component score $(\mathrm{P}=0.001)$. Who well-being had no correlation with physical functioning, role physical of quality of life domains.

According to who well-being score $16.50 \%$ of patients scored between (29-50) and $83.50 \%$ of patients scored between (51-100). Although we found some unexpected associations. For example, who well being score reported less in the age group of (18-25) and (55-65) [Table 5].

Complete DTSQ scores were available for 103 patients. Given the maximum possible score of 36, satisfaction was high (mean 32.74, SEM 0.21). Presence of co morbidities, patients on insulin therapy and (18-35) age group patients were less satisfied with the treatment than other patients [Table 6].

\section{Figure:1}

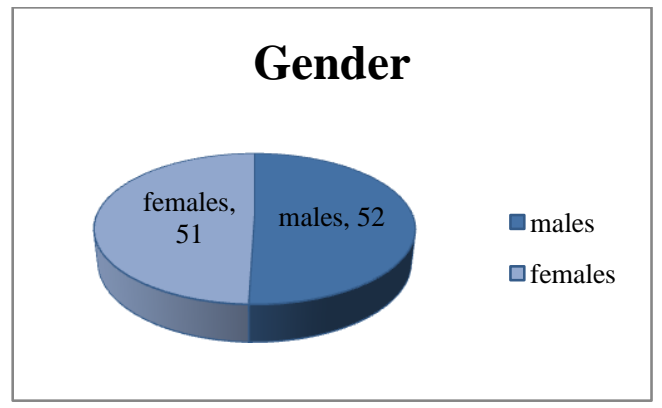

\section{Figure:2}

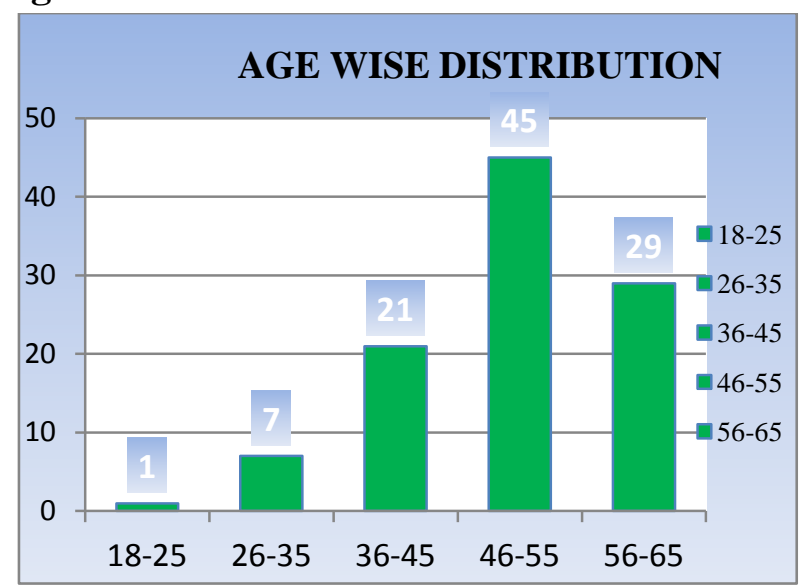




\section{JMSCR Vol||05||Issue||09||Page 28274-28283||September}

Figure: 3

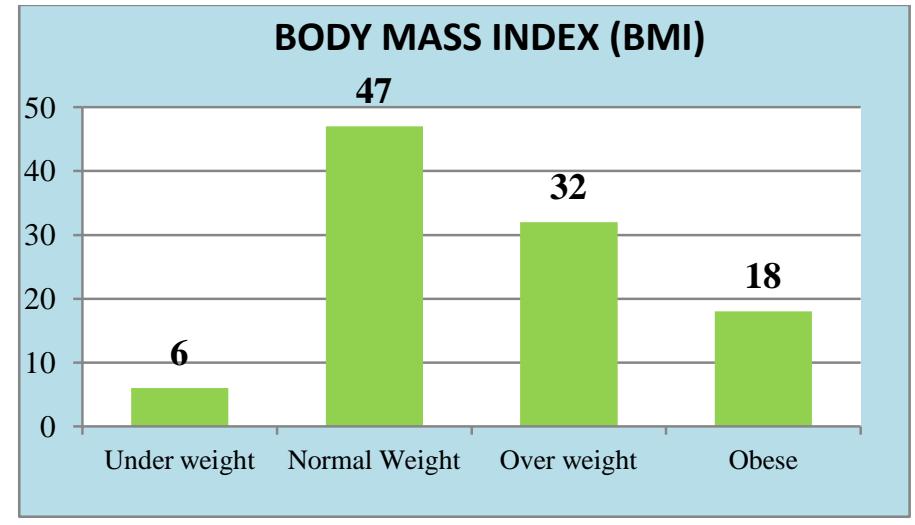

Figure: 4

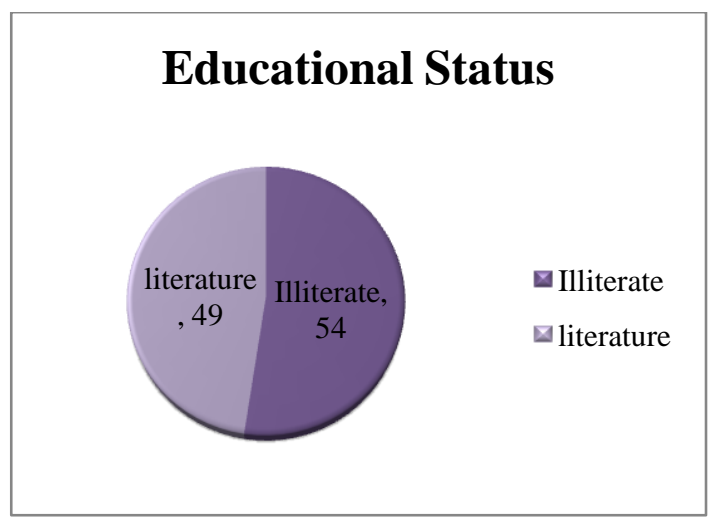

Figure: 5

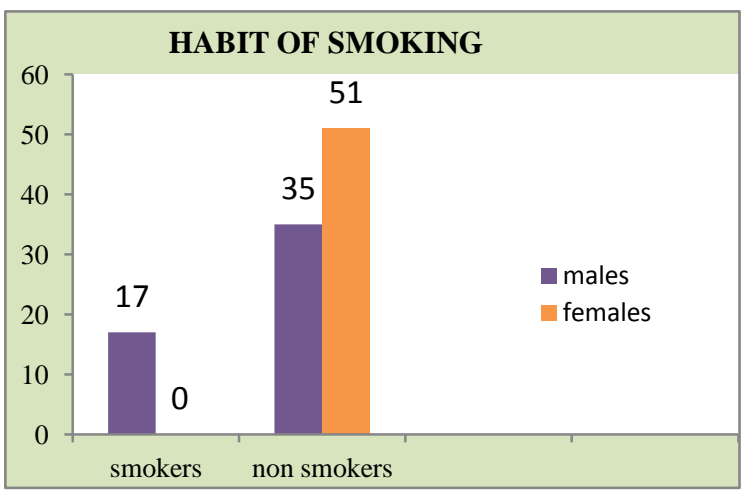

Figure: 6

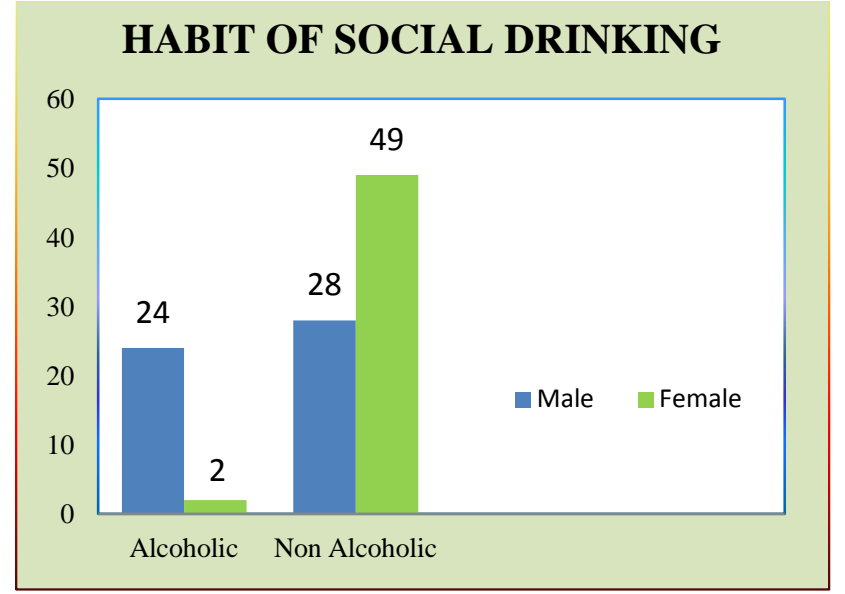

Figure: 7

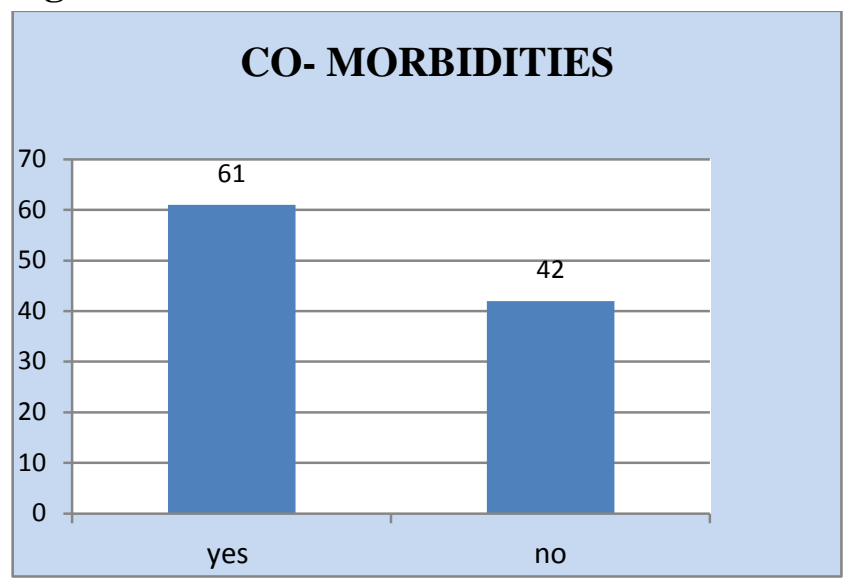

Figure: 8

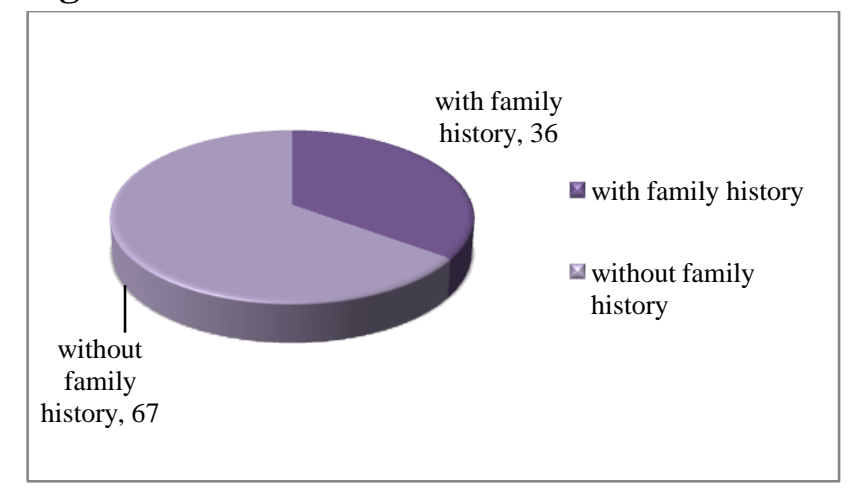

Figure: 9

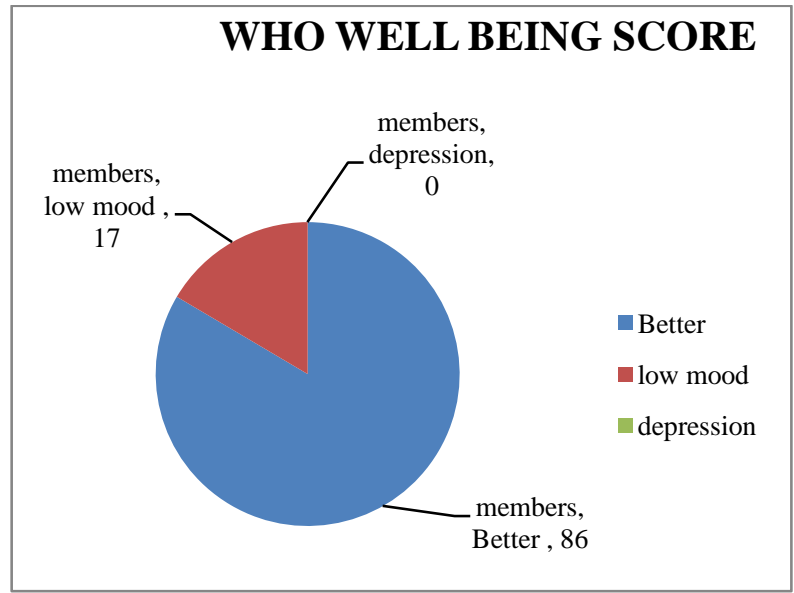

Figure: 10

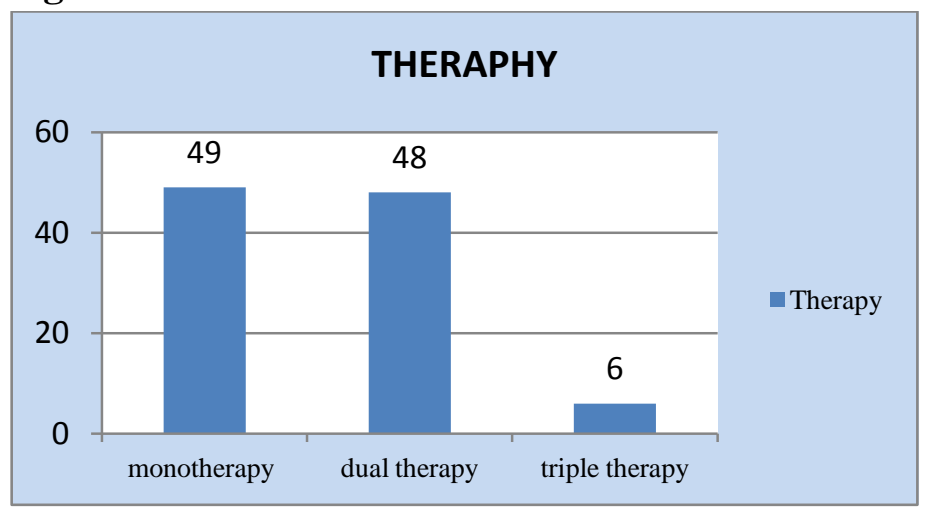


Figure: 11 Occupation Status

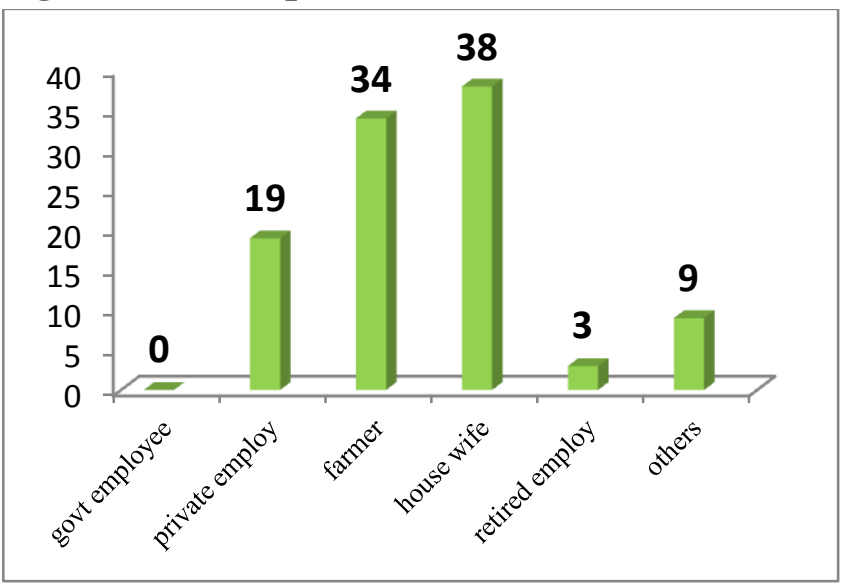

Figure: 12

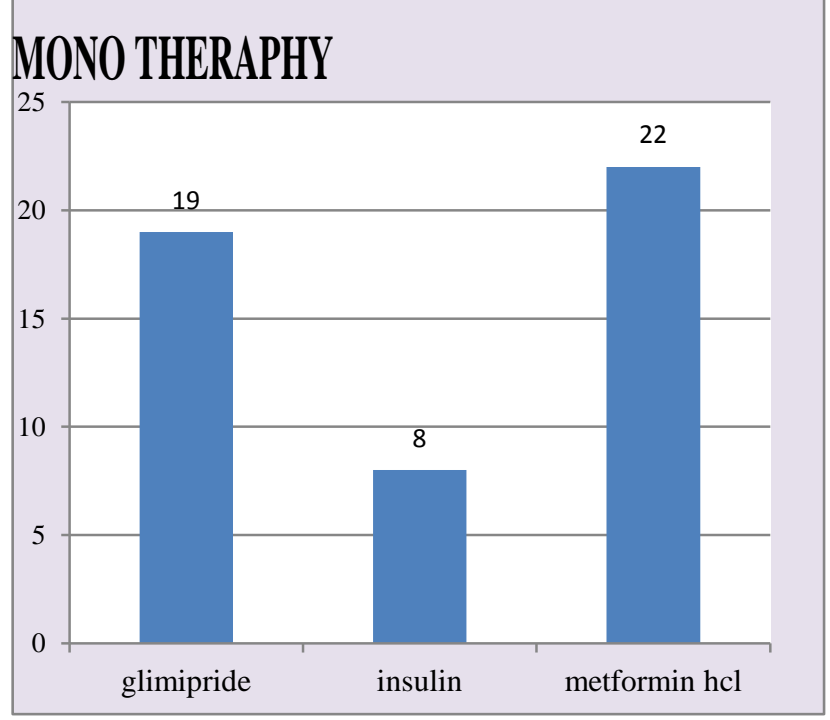

Table 1: Characteristics of the sample $(N=103)$ of type 2 diabetics

\begin{tabular}{|c|c|c|}
\hline $\begin{array}{l}\text { Variable } \\
\%\end{array}$ & $\mathrm{~N}$ & \\
\hline \multicolumn{3}{|l|}{ Gender } \\
\hline Male & 52 & 50.5 \\
\hline Female & 51 & 49.5 \\
\hline \multicolumn{3}{|l|}{ Age group } \\
\hline $18-25$ & 1 & 0.9 \\
\hline $26-35$ & 7 & 6.8 \\
\hline $36-45$ & 21 & 20.4 \\
\hline $46-55$ & 45 & 43.7 \\
\hline $56-65$ & 29 & 28.2 \\
\hline \multicolumn{3}{|l|}{ BMI } \\
\hline$\leq 18$ & 6 & 5.8 \\
\hline $19-24$ & 47 & 45.6 \\
\hline $25-29$ & 32 & 31.1 \\
\hline$\geq 30$ & 18 & 17.5 \\
\hline \multicolumn{3}{|l|}{ Education } \\
\hline Illiterate & 54 & 52.4 \\
\hline Literate & 49 & 47.6 \\
\hline \multicolumn{3}{|l|}{ Smoker } \\
\hline Yes & 17 & 16.5 \\
\hline No & 86 & 83.5 \\
\hline \multicolumn{3}{|l|}{ Alcoholic } \\
\hline Yes & 26 & 25.2 \\
\hline No & 77 & 74.8 \\
\hline \multicolumn{3}{|l|}{ Co morbidity } \\
\hline Yes & 61 & 59.2 \\
\hline No & 42 & 40.8 \\
\hline \multicolumn{3}{|l|}{ Family history } \\
\hline Yes & 36 & 35.0 \\
\hline No & 67 & 65.0 \\
\hline \multicolumn{3}{|l|}{ Who-well being score } \\
\hline Better $(51-00)$ & 86 & 83.5 \\
\hline Low $\operatorname{mood}(29-50)$ & 17 & 16.5 \\
\hline \multicolumn{3}{|l|}{ Therapy } \\
\hline Mono therapy & 49 & 47.6 \\
\hline Dual therapy & 48 & 46.6 \\
\hline
\end{tabular}


Triple therapy

Occupation status

Govt employee

Private employee

Farmer

House wife

Retired employee

Others

Mono therapy drugs $(N=49)$

Glimipride

Metformin hcl

Insulin
6

0

19

34

38

3

9

19

22

8
5.8

0

18.4

33.1

36.9

2.9

8.7

38.8

44.9

16.3

Table 2: Domain score in both gender.

\begin{tabular}{lcc}
\hline Domains & Mean & SEM \\
\hline Physical functioning & 53.96 & 2.32 \\
Role physical & 25.00 & 3.79 \\
Bodily pain & 60.31 & 2.83 \\
General health & 53.79 & 1.88 \\
Vitality & 55.02 & 1.93 \\
Social functioning & 71.50 & 1.97 \\
Role emotional & 60.92 & 1.96 \\
Mental health & 41.10 & 4.46
\end{tabular}

Table 3 : Mean, Sem, P-Value of SF-36 QOL scores according to the various variables.

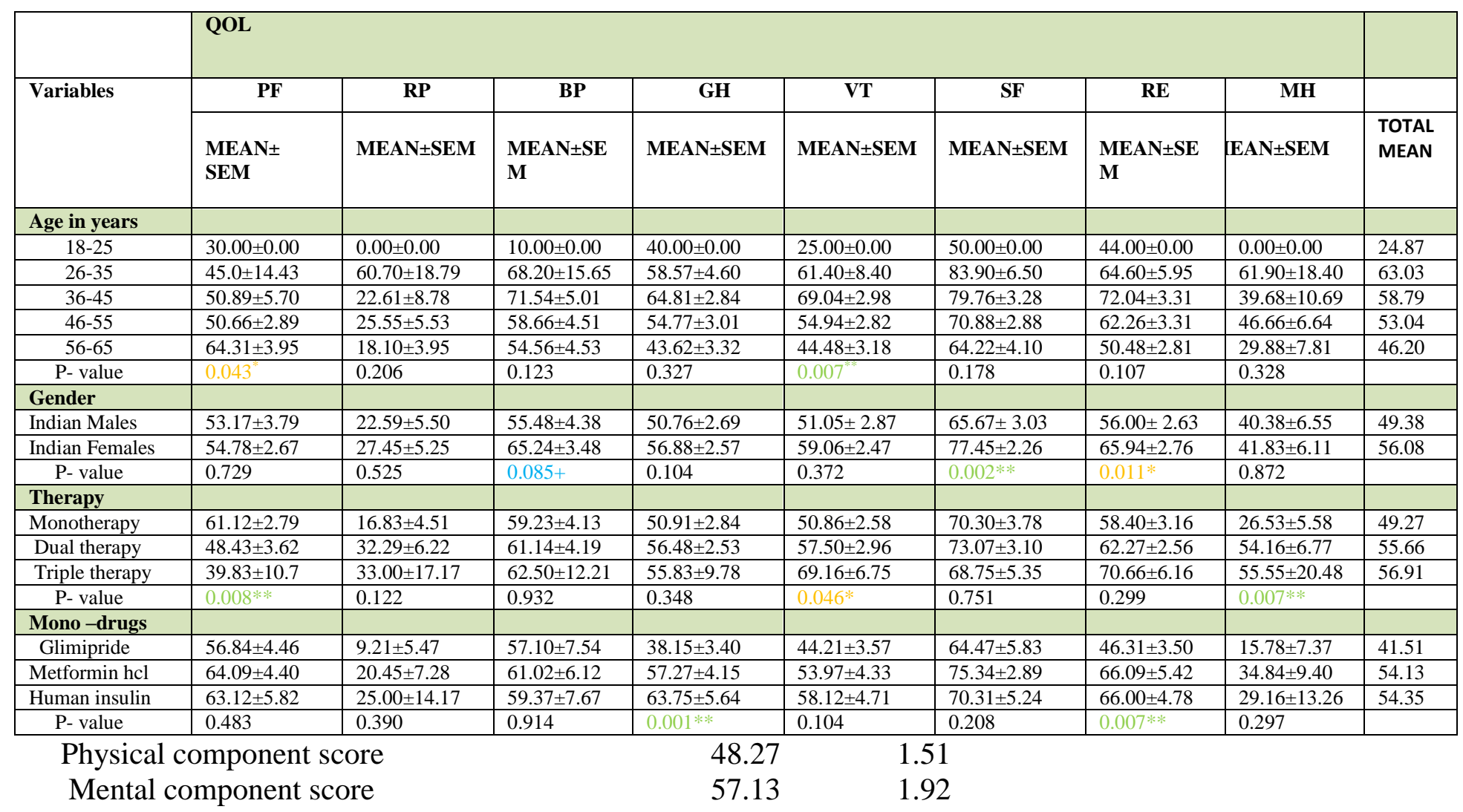




\begin{tabular}{|c|c|c|c|c|c|c|c|c|c|}
\hline BMI $\left(\mathrm{kg} / \mathrm{m}^{2}\right)$ & & & & & & & & & \\
\hline$\leq 18.5$ & $60.00 \pm 8.85$ & $33.33 \pm 17.85$ & $64.58 \pm 8.25$ & $58.33 \pm 8.02$ & $61.66 \pm 6.54$ & $81.25 \pm 8.38$ & $68.00 \pm 7.65$ & $61.11 \pm 18.08$ & 61.03 \\
\hline $18.6-24.9$ & $52.12 \pm 3.57$ & $23.40 \pm 5.17$ & $67.76 \pm 3.67$ & $60.42 \pm 2.31$ & $60.05 \pm 2.59$ & $74.46 \pm 2.56$ & $64.21 \pm 2.61$ & $60.05 \pm 2.59$ & 57.80 \\
\hline $25-29.9$ & $54.25 \pm 4.29$ & $25.00 \pm 7.36$ & $50.07 \pm 5.42$ & $46.09 \pm 3.70$ & $47.96 \pm 3.80$ & $65.46 \pm 3.61$ & $54.12 \pm 3.29$ & $39.58 \pm 8.52$ & 47.81 \\
\hline$\geq 30$ & $56.04 \pm 5.05$ & $26.38 \pm 9.79$ & $57.30 \pm 7 \pm 65$ & $48.67 \pm 4.24$ & $52.22 \pm 4.39$ & $71.25 \pm 5.58$ & $62.05 \pm 5.95$ & $46.29 \pm 11.15$ & 52.52 \\
\hline P-value & 0.854 & 0.945 & $0.056+$ & $0.005 * *$ & $0.038 *$ & 0.148 & 0.120 & 0.637 & \\
\hline \multicolumn{10}{|l|}{ Smoker } \\
\hline Yes & $52.64 \pm 6.30$ & $29.41 \pm 10.33$ & $43.09 \pm 7.69$ & $49.41 \pm 5.37$ & $52.05 \pm 3.61$ & $61.91 \pm 6.38$ & $56.23 \pm 3.11$ & $54.90 \pm 11.41$ & 49.95 \\
\hline No & $54.32 \pm 2.50$ & $24.12 \pm 4.08$ & $63.72 \pm 2.92$ & $54.66 \pm 1.99$ & $55.61 \pm 2.20$ & $73.40 \pm 1.96$ & $61.48 \pm 2.26$ & $38.37 \pm 4.82$ & 53.21 \\
\hline P-value & 0.817 & 0.639 & $0.021 *$ & 0.370 & 0.408 & 0.102 & 0.154 & 0.196 & \\
\hline \multicolumn{10}{|l|}{ Alcoholic } \\
\hline Yes & $47.30 \pm 4.56$ & $21.15 \pm 7.92$ & $45.09 \pm 6.53$ & $44.80 \pm 4.11$ & $48.84 \pm 3.43$ & $60.19 \pm 4.33$ & $54.46 \pm 3.04$ & $42.30 \pm 9.52$ & 45.51 \\
\hline No & $56.22 \pm 2.66$ & $26.29 \pm 4.33$ & $65.45 \pm 2.88$ & $56.83 \pm 1.99$ & $57.11 \pm 2.27$ & $75.32 \pm 2.04$ & $63.10 \pm 2.37$ & $40.69 \pm 5.06$ & 55.11 \\
\hline P-value & $0.099+$ & 0.572 & $0.007 * *$ & $0.012 *$ & $0.050 *$ & $0.003 * *$ & $0.029 *$ & 0.882 & \\
\hline \multicolumn{10}{|c|}{\begin{tabular}{|l|l|} 
Comorbidities & \\
\end{tabular}} \\
\hline Yes & $\begin{array}{l}57.11 \pm \\
2.75\end{array}$ & $20.49 \pm 4.35$ & $63.07 \pm 3.15$ & $53.60 \pm 2.10$ & $53.36 \pm 2.30$ & $\begin{array}{l}71.06 \pm \\
2.35\end{array}$ & $58.52 \pm 2.22$ & $36.06 \pm 5.35$ & 51.65 \\
\hline No & $49.40 \pm 3.98$ & $31.54 \pm 6.76$ & $56.30 \pm 5.21$ & $54.07 \pm 3.48$ & $57.44 \pm 3.36$ & $72.14 \pm 3.46$ & $64.40 \pm 3.53$ & $48.41 \pm 7.63$ & 54.21 \\
\hline P-value & 0.116 & 0.174 & 0.271 & 0.909 & 0.320 & 0.798 & 0.164 & 0.190 & \\
\hline \multicolumn{10}{|l|}{ Well being } \\
\hline Better 51-100 & $53.88 \pm 2.49$ & $26.45 \pm 4.20$ & $65.78 \pm 2.79$ & $57.57 \pm 1.89$ & $57.70 \pm 2.02$ & $75.23 \pm 1.84$ & $63.21 \pm 2.10$ & $45.34 \pm 4.89$ & 55.69 \\
\hline $\begin{array}{l}\text { Low mood 29- } \\
50\end{array}$ & $54.41 \pm 6.34$ & $17.64 \pm 8.77$ & $32.64 \pm 6.57$ & $34.70 \pm 3.54$ & $41.47 \pm 4.49$ & $52.64 \pm 5.74$ & $47.29 \pm 3.94$ & $19.60 \pm 9.50$ & 37.54 \\
\hline $\mathrm{P}$-value & 0.934 & 0.392 & $0.000^{* * * *}$ & $0.000^{* * * *}$ & 0.003 ** & $0.001^{\prime}$ & $0.001^{\prime \prime}$ & $0.024 *$ & \\
\hline
\end{tabular}

\begin{tabular}{|c|l|l|l|l|l|l|l|l|l|}
\hline Education & & & & & & & & \\
\hline Illiterate & $59.23 \pm 3.17$ & $25.92 \pm 5.16$ & $54.02 \pm 3.83$ & $51.57 \pm 2.67$ & $50.09 \pm 2.42$ & $67.91 \pm 2.91$ & $55.44 \pm 2.41$ & $35.80 \pm 5.63$ & 49.99 \\
\hline Literature & $48.16 \pm 3.22$ & $23.98 \pm 5.64$ & $67.24 \pm 4.01$ & $56.24 \pm 2.61$ & $60.45 \pm 2.89$ & $75.45 \pm 2.54$ & $66.95 \pm 2.94$ & $46.93 \pm 7.00$ & 55.67 \\
\hline P- value & 0.218 & $0.016^{*}$ & 0.800 & $0.019 *$ & 0.215 & $0.007 * *$ & $0.054+$ & $0.003 * *$ & \\
\hline
\end{tabular}

Above table shows the comparative distribution of individual QOL domain scores (i:e PE $\pm \mathrm{RP} \pm \mathrm{BP} \pm \mathrm{GH} \pm$ $\mathrm{VT} \pm \mathrm{SF} \pm \mathrm{RE} \pm$ and $\mathrm{MH}$ ) of patient with respect to the various variables.
$<0.001 * * *$ extremely significant
$0.001-0.01 * *$ strongly significant
$0.01-0.05 *$ moderately significant
$0.05-0.10+$ slightly significant
$>0.10$ not significant

Table 4: Mean, Sem, P-Value of PCS and MCS scores according to various variables.

\begin{tabular}{|c|c|c|}
\hline \multirow{2}{*}{ VARIABLES } & QOL \\
\cline { 2 - 3 } & PCS & MCS \\
\cline { 2 - 3 } Age in years & & \\
\hline $18-25$ & & \\
\hline $26-35$ & $20.00 \pm 0.00$ & $29.75 \pm 0.00$ \\
\hline $36-45$ & $58.12 \pm 8.33$ & $67.95 \pm 8.79$ \\
\hline $46-55$ & $52.47 \pm 3.07$ & $65.13 \pm 3.89$ \\
\hline $56-65$ & $47.41 \pm 2.29$ & $58.69 \pm 2.95$ \\
\hline P- value & $45.15 \pm 2.38$ & $47.26 \pm 2.72$ \\
\hline Gender & $0.054+$ & $0.003 * *$ \\
\hline Male & & \\
\hline Female & $45.50 \pm 2.44$ & $53.32 \pm 3.00$ \\
\hline P- value & $51.09 \pm 1.70$ & $61.08 \pm 2.28$ \\
\hline Therapy & $0.064+$ & $0.042 *$ \\
\hline
\end{tabular}




\begin{tabular}{|c|c|c|}
\hline Monotherapy & $47.02 \pm 1.97$ & $51.52 \pm 2.40$ \\
\hline Dual therapy & $49.58 \pm 2.38$ & $61.75 \pm 3.03$ \\
\hline Triple therapy & $47.87 \pm 7.86$ & $66.03 \pm 6.62$ \\
\hline P-value & 0.717 & $0.017 *$ \\
\hline \multicolumn{3}{|l|}{ MonoTherapy drugs } \\
\hline glimipride & $40.32 \pm 3.50$ & $42.69 \pm 3.83$ \\
\hline Metformin hcl & $50.71 \pm 2.54$ & $57.56 \pm 3.52$ \\
\hline Human insulin & $52.81 \pm 3.23$ & $55.90 \pm 2.57$ \\
\hline P-value & $0.021 *$ & $0.011 *$ \\
\hline \multicolumn{3}{|l|}{ BMI $\left(\mathrm{kg} / \mathrm{m}^{2}\right)$} \\
\hline$\leq 18.5$ & $54.06 \pm 4.27$ & $68.00 \pm 8.93$ \\
\hline $18.6-24.9$ & $50.95 \pm 1.71$ & $59.08 \pm 2.57$ \\
\hline $25-29.9$ & $43.85 \pm 3.34$ & $57.78 \pm 3.55$ \\
\hline$\geq 30$ & $47.18 \pm 4.00$ & $57.95 \pm 5.02$ \\
\hline $\mathrm{P}$ - value & 0.171 & 0.190 \\
\hline \multicolumn{3}{|l|}{ Smoker } \\
\hline Yes & $40.17 \pm 3.56$ & $56.27 \pm 5.37$ \\
\hline No & $49.18 \pm 1.52$ & $57.30 \pm 2.05$ \\
\hline $\mathrm{P}$-value & $0.030 *$ & 0.860 \\
\hline \multicolumn{3}{|l|}{ Alcoholic } \\
\hline Yes & $39.59 \pm 4.17$ & $51.45 \pm 4.42$ \\
\hline No & $51.20 \pm 1.31$ & $59.05 \pm 2.06$ \\
\hline $\mathrm{P}$-value & $0.013^{*}$ & 0.128 \\
\hline \multicolumn{3}{|l|}{ Co morbidities } \\
\hline Yes & $48.57 \pm 1.48$ & $54.75 \pm 2.08$ \\
\hline No & $47.83 \pm 3.05$ & $60.60 \pm 3.57$ \\
\hline P-value & 0.829 & 0.163 \\
\hline \multicolumn{3}{|l|}{ Well being } \\
\hline Better (51-100) & $50.92 \pm 1.46$ & $34.85 \pm 4.13$ \\
\hline Low mood (29-50) & $60.47 \pm 1.93$ & $40.25 \pm 4.58$ \\
\hline P-value & $0.002 * *$ & $0.001 * *$ \\
\hline \multicolumn{3}{|l|}{ Education } \\
\hline Illiterate & $47.69 \pm 2.07$ & $52.31 \pm 2.37$ \\
\hline Literature & $48.90 \pm 2.23$ & $62.45 \pm 2.91$ \\
\hline P-value & 0.690 & $0.008 * *$ \\
\hline
\end{tabular}

Above table shows the comparison of PCS and MCS scores with the various Variables.

Table 5: Who-Well Being Score according to age wise distribution.

\begin{tabular}{|c|c|}
\hline Age in years & Well being score \\
\hline $18-25$ & 44 \\
\hline $26-35$ & 73.14 \\
\hline $36-45$ & 90 \\
\hline $46-55$ & 78.62 \\
\hline $56-65$ & 70.13 \\
\hline
\end{tabular}

Table 6: DTSQ scores:

\begin{tabular}{|l|c|}
\hline \multicolumn{1}{|c|}{ Variables } & DTSQ Score \\
\hline Over all & $32.74 \pm 0.21$ \\
\hline $18-25$ age & 28 \\
\hline $26-35$ age & $29.50 \pm 0.37$ \\
\hline Insulin & $30.75 \pm 0.25$ \\
\hline Co-morbidities & $32.01 \pm 0.17$ \\
\hline
\end{tabular}




\section{Discussion}

Totally, type 2 diabetics of our study had lower quality of life in diverse domains in comparison with similar studies. Our research indicated that quality of life decreases with age which is similar to previous studies carried out on diabetic patients $[10,11,12,13,1,4,15]$. The patients with type 2 DM had better mental component score than physical component score which was similar to previous studies carried out on diabetic patients ${ }^{[16]}$. Male gender had a lower quality of life than females which was opposite to other studies carried ${ }^{[10,11,}$

${ }^{15]}$. Our study reported that WHO well-being score was lesser in the age group of (18-25) and (56-65). These results are in accordance with other studies carried out on diabetic patients ${ }^{[10]}$. Our research showed that age variable had meaningful reverse correlation with physical functioning, vitality, physical component score, and mental component score domains. A direct correlation was found between age and physical functioning in ${ }^{[17]}$. Our research showed that gender variable correlated to bodily pain, social functioning, role emotional, physical component score, mental component score. Earlier author ${ }^{[17]}$ observed a relationship between gender and all quality of life domains as men's scores was higher in all domains. We found it out that education level has a direct relationship with role physical, general health, social functioning, role emotional, mental health, and mental component score. Some authors observed a direct relationship between education level and all quality of life domains other than general health and role emotional ${ }^{[17]}$.

\section{Conclusion}

Diabetic patients perceive a decrease in health related quality of life (HRQOL) as their age increases. High health related quality of life (HRQOL) represents an ultimate goal and important outcomes of all medical intervention in diabetic patients. Factors related to lower (HRQOL) include presence of obesity, smoking, alcoholic, illiterate and low mood. Men had lower well being score than women. Improving of
(HRQOL) by appropriate education and follow-up must be emphasized to the management of diabetic patients. Clinical and (QOL) instruments should be used together to get an appropriate overview of the health status of patients with diabetes and QOL measures should be routinely employed in clinical, research, population and policy-related situation. Individualized care of patients with diabetes should be considered for improving the QOL.The public health concern about the increasing number of people with diabetes should be addressed especially in people with impaired HRQOL. Taken together, the findings of these population studies indicate that people using either pharmacological therapy or lifestyle modification to treat diabetes rated their physical health as poor.

\section{References}

1. https://www.staff.ncl.ac.uk/philip.home/w ho_dmg.pdf

2. Kamel NM, Badawy YA, El-Zeiny NA, Merdan IA. Behaviour of patients in relation to management of their disease. Eastern Mediterr Health J 1999;5:967-73.

3. King $\mathrm{H}$. WHO and the International Diabetes federation: regional Partners. Bull World Health Organ 1999;77:954.

4. Tankova T, Dakovska G, Koev D. Education of diabetic Patients - a one year experience. Patient Educ Couns 2001;43:139-45.

5. www.who.int/mental_health/media/68.pdf

6. www.healthypeople.gov/2020/about/found ation-health-measures/health-relatedquality-of-life-and-well-being.com.

7. www.health.wa.gov.au/publications/docu ments/chronic\%20disease.pdf.

8. www.bjjprocs.boneandjoint.org.uk/content /90-B/SUPP_III/448.1.short.

9. www.dawnstudy.com.

10. W. Ken redekop, marc.A. koopmanschap, Ronald p. Stolk, Louis w. Niessen. healthrelated quality of life and treatment 
satisfaction in dutch patients with type2diabetes.diabetic care. 2002 25. 458-463.

11. Morales MC, Navas AF, Jimenez MFR, Ramos JMR (2015) Health-Related Quality of Life in Patients with Type 2 Diabetes Mellitus in a RuralArea. J Diabetes Metab 6: 572.

12. Alonso J, Prieto L, Antó JM (1995) [The Spanish version of the SF-36 Health Survey (the SF-36 health questionnaire): an instrument for measuring clinical results]. Med Clin (Barc) 104: 771-776.

13. Espinosa De Los Monteros MJ, Alonso J, Ancochea J, González A (2002) Quality of life in asthma: reliability and validity of the short form generic questionnaire (SF36) applied to the population of asthmatics in a public health area. Arch Bronconeumol 38: 4-9.

14. Ambriz Y, Menor R, Campos-González I D y Cardiel M H (2015) Calidad de vida relacionada con la salud en artritis reumatoide, osteoartritis, diabetes mellitus, insuficiencia renal terminal y población geriátrica. Experiencia de un Hospital General en México. Reumatología Clínica 11: 68-72.

15. Ana spasic, radmila velickovic, Aleksandra catic dordevic, nikola stefanovic, Quality of life in type diabetic patients. Scientific journel of the faculty of medicine in nis:2014:31(3); 193-200.

16. M. H. Kazemi-galougahi, H. Navidi ghazian, H. eftekhar ardebili, M. Mahmoudi.quality of life in type diabetic patients and related effective factors:Indian journel of medical sciences: 66: 230-235.

17. Papadopoulos AA, Kontodimopoulos N, Frydas A, Ikonomakis E, Niakas D. Predictors of health-related quality of life in type II diabetic patients in Greece. BMC Public health 2007: 7:186. 